\section{Sickly channels in mild disease}

\section{Christopher Miller}

From the intellectual viewpoint, cystic fibrosis (CF) rivals sickle-cell anaemia as the prime example of a heuristically rich molecular disease - it can be viewed all the way from bottom to top, from the primary error in DNA sequence to the clinical manifestations. The genetic error results in defective epithelial $\mathrm{Cl}^{-}$ion channels essential to fluid secretion, and a small army of researchers is at work on identifying just what the functional consequences are for the channel of naturally occurring mutations of the CF gene. On page 160 of this issue ${ }^{1}$, Sheppard and colleagues present such a sequence-tofunction mapping for several of the gene's alleles.

Our molecular understanding of CF, a devastating disease, has emerged with amazing speed. Only five years ago the causative lesion was unknown, even at the level of cell physiology, although defective epithelial transport of $\mathrm{Cl}^{-}$was widely suspected to be the culprit. Then, in 1989 , the altered gene was cloned ${ }^{2,3}$ and its product named the cystic fibrosis transmembrane regulator (CFTR) because of its 12 putative membranespanning $\alpha$-helical stretches (see figure). A robust debate immediately ensued about the precise function of the CFTR. Is it indeed an epithelial $\mathrm{Cl}^{-}$channel, or perhaps a protein involved in the delivery of $\mathrm{Cl}^{-}$channels to the plasma membrane? The question now seems settled: CFTR is a $\mathrm{Cl}^{-}$channel. This assertion would have elicited uproar only a year ago, but now two lines of evidence make it hard to avoid. First, certain point mutations in the CFTR gene (transfected into mammalian cells and assayed by patch recording) alter the interanionic selectivity of the open channel without affecting the regulation of its gating by phosphorylation and $\mathrm{ATP}^{4}$. Second, the CFTR protein, heterologously expressed and purified (in the denaturing detergent SDS, no less), induces $\mathrm{Cl}^{-}$channels with the correct properties when reconstituted into 'artificial' planar lipid bilayer membranes 5 .

These electrophysiological assays set the stage for a detailed examination of the behaviour of channels arising in commonly occurring CFTR mutants. About $70 \%$ of $\mathrm{CF}$ patients carry the same mutation, a single amino-acid deletion $(\Delta F 508)$ in one of the two nucleotide-binding domains (see figure). This mutation results in a temperaturesensitive defect in protein processing (not aberrant regulation by ATP, as might have been expected); at $27^{\circ} \mathrm{C}$, the mutant CFTR forms functionally active channels, but at $37^{\circ} \mathrm{C}$, it gets stuck in the maturation pathway and fails to reach the plasma membrane ${ }^{6}$, thus giving rise to a very severe disease state in which the epithelial $\mathrm{Cl}^{-}$channels are absent.

The report on page 160 examines three less common mutations (affecting about $2 \%$ of $\mathrm{CF}$ patients) that lead to mild forms of the disease. Each of these missense mutations alters a different arginine residue, one in the second transmembrane helix (M2), and two in the sixth (M6). When CFTR complementary DNAs bearing these individual mutations are transfected into mammalian epithelial cells, appropriately regulated $\mathrm{Cl}^{-}$channels appear in the plasma membrane. However, the absolute amount of $\mathrm{Cl}^{-}$current per cell is abnormally low, $5-30 \%$ of that induced by wild-type CFTR. Single-channel analysis revealed the reasons for this diminished whole-cell current. The two charge-neutralized M6 mutants, R334W and R347P, show a lowered singlechannel conductance implying an altered $\mathrm{Cl}^{-}$conduction pathway. The M2 mutant $\mathrm{R} 117 \mathrm{H}$, activated as usual by phosphorylation, displays a unitary conductance only slightly lower than normal, but its probability of being open is vastly reduced.

These different types of alteration in single-channel properties nicely account for the low whole-cell currents seen in the mutants, and they rationalize the mild nature of the disease in which the patient retains significant $\mathrm{Cl}^{-}$transport function. The results also focus mechanistic attention on $\mathrm{M} 2$ and M6, highlighting how nature has already constructed point mutations affecting both gating and open-channel conduction properties.
More than 300 CFTR mutant alleles have been identified by genetic screening so far, and they provide a fertile field, already under cultivation, from which to harvest insight into the molecular mechanism of this crucial ion channel.

Cystic fibrosis is prevalent in Europe and North America, about 5\% of Caucasians being heterozygous for deleterious forms of the gene. An obvious question arising from the disease aetiology is why the clinically severest mutant CFTR gene - effectively a null phenotype - is so much more prevalent in the population than the less harmful variants. Why, indeed, has the $\triangle F 508$ mutation spread

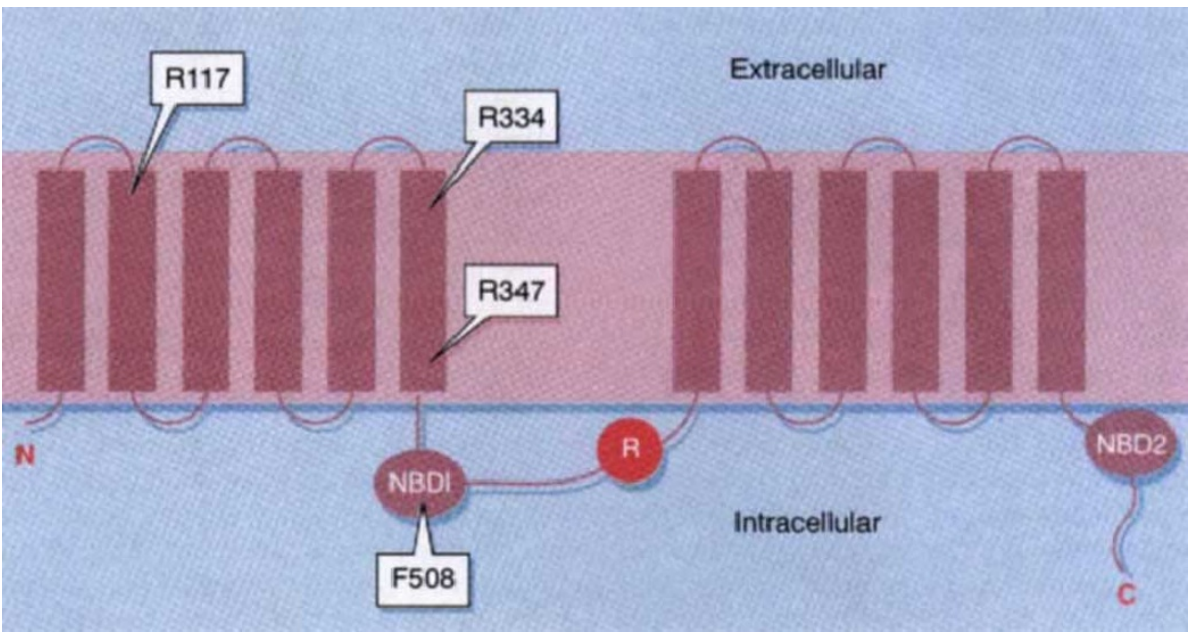

Positions of the mutations in the CFTR protein discussed in the text. NBD1 and NBD2, nucleotide-binding domains. $\mathrm{R}$, regulatory phosphorylation domain.

so quickly through the population in less than 10,000 years $^{7}$ ? Questions such as this invite speculation as to the possible selective advantage conferred upon $\mathrm{CF}$ heterozygous individuals, and the field is replete with untested guesses ${ }^{7}$. My favourite, offered by Baxter et al. ${ }^{8}$, is that a deficiency in epithelial fluid secretion (perhaps subtly present in heterozygotes) would ameliorate the severity of enterotoxin-elicited diarrhoea, which is by far the most important cause of infant mortality in primitive societies - susceptibility to lethal dehydration has probably been with our species throughout our evolutionary history and could account for a protective effect of this recent mutation.

Christopher Miller is in the Howard Hughes Medical Institute, Graduate Department of Biochemistry, Brandeis University, Waltham, Massachusetts 02254, USA.

1. Sheppard, D. N. et al. Nature 362, 160-164 (1993). 2. Riordan, J. R. et al. Science 245, 1066-1073 (1989) 3. Rommens, J. M. et at. Science 245, 1059-1065 (1989) 4. Anderson, M. P. et al. Science 253, 202-205 (1991). 5. Bear, C. et al. Cell 68, 809-818 (1992)

6. Denning, G. M. et al. Nature 358, 761-764 (1992)

7. Tsui, L.-C. \& Buchald, M. Adv. hum. Genet. 20, 153-267 (1991).

8. Baxter, P. S., Goldhill, J., Hardcastle, J., Hardcastle, P. T. \& Taylor, C. J. Nature 335, 211 (1988)

NATURE - VOL 362 - $11 \mathrm{MARCH} 1993$ 